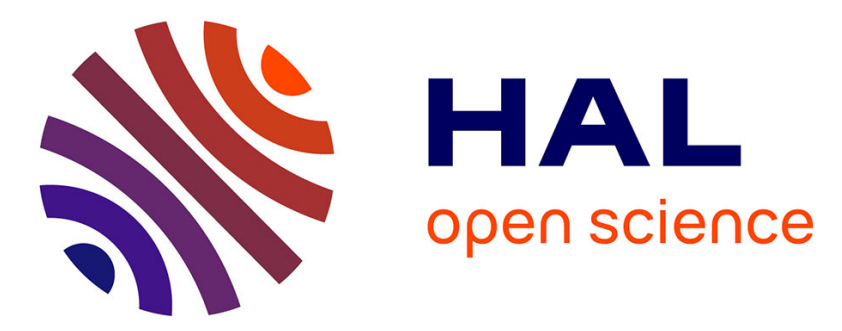

\title{
Broadband sub-diffraction and ultra-high energy density focusing of elastic waves in planar gradient-index lenses
}

Jinfeng Zhao, Xiaodong Cui, Bernard Bonello, Bahram Djafari-Rouhani, Weitao Yuan, Yongdong P Pan, Jie Ren, Xiaoqing Zhang, Zheng Zhong

\section{- To cite this version:}

Jinfeng Zhao, Xiaodong Cui, Bernard Bonello, Bahram Djafari-Rouhani, Weitao Yuan, et al.. Broadband sub-diffraction and ultra-high energy density focusing of elastic waves in planar gradient-index lenses. Journal of the Mechanics and Physics of Solids, 2021, 150, pp.104357. 10.1016/j.jmps.2021.104357 . hal-03141945

\section{HAL Id: hal-03141945 \\ https://hal.sorbonne-universite.fr/hal-03141945}

Submitted on 15 Feb 2021

HAL is a multi-disciplinary open access archive for the deposit and dissemination of scientific research documents, whether they are published or not. The documents may come from teaching and research institutions in France or abroad, or from public or private research centers.
L'archive ouverte pluridisciplinaire HAL, est destinée au dépôt et à la diffusion de documents scientifiques de niveau recherche, publiés ou non, émanant des établissements d'enseignement et de recherche français ou étrangers, des laboratoires publics ou privés. 


\title{
Broadband sub-diffraction and ultra-high energy density focusing of elastic waves in planar gradient-index lenses
}

\author{
Jinfeng Zhao ${ }^{1}$, Xiaodong Cui ${ }^{1}$, Bernard Bonello ${ }^{2}$, Bahram Djafari-Rouhani ${ }^{3}$, Weitao Yuan ${ }^{1}$, Yongdong Pan $^{1}$, Jie \\ Ren $^{4}$, Xiaoqing Zhang ${ }^{4}$, and Zheng Zhong ${ }^{1,5 *}$ \\ ${ }^{1}$ School of Aerospace Engineering and Applied Mechanics, Tongji University, 100 Zhangwu Road, 200092 Shanghai, China \\ ${ }^{2}$ Sorbonne Université, UPMC Université Paris 06, INSP UMR CNRS 7588, 4 Place Jussieu, 75005 Paris, France \\ ${ }^{3}$ Institut d'Electronique, de Microélectronique et de Nanotechnologie (IEMN-UMR CNRS 8520), Université de Lille Sciences et \\ Technologies, Cité Scientifique, 59652 Villeneuve d'Ascq Cedex, France \\ ${ }^{4}$ Shanghai Key Laboratory of Special Artificial Microstructure Materials and Technology, School of Physics Sciences and \\ Engineering, Tongji University, 1239 Siping Road, 200092 Shanghai, China \\ ${ }^{5}$ School of Science, Harbin Institute of Technology, Shenzhen, 518055, PR, China
}

\begin{abstract}
We demonstrate experimentally and theoretically the broadband sub-diffraction and ultra-high energy density focusing of elastic wave inside planar gradient-index (GRIN) plate lenses based on thickness variation. The full width at half maximum is $\sim 0.40$ times the minimum wavelength inside the lens $\lambda_{0}$ or $\sim 1 / 11$ times the incident wavelength in the background plate $\lambda_{\mathrm{B}}$. We analytically elucidate the underlying physical mechanism as the rapid oscillations in the propagator function at the focal distance. Our design displays several advantages including the broadband sub-diffraction behavior, three-order of magnitudes higher energy intensity at the focal point, and focusing capacity with a narrow transverse width of the lens, over the usual metastructure and/or phononic crystal based GRIN lenses. The broadband sub-diffraction and ultra-high energy density focusing can be used for energy harvesting and signal sensing.
\end{abstract}

Keywords: gradient-index lens, sub-diffraction focusing, metamaterials, energy harvesting

\section{Introduction}

Gathering elastic wave energy or confining phonons over a sub-diffraction spot in a broad frequency range is fascinating, not only because of various applications, but also due to the lack in nature of materials having this property. To meet this challenge, a great deal of works has been devoted to the realization of structures and devices designed to focus wave beams over a sub-diffraction spot typically featuring the full width at half maximum (FWHM) less than half the wavelength. A first category inspired by J.B. Pendry's seminal work (Pendry, 2000), relies on evanescent waves in the near field as evidenced in negative-refraction lenses (Sukhovich et al., 2009; Zhou and Hu, 2011; Zhao et al., 2016b; Dong et al., 2017), hyperlenses (Lu and Liu, 2012), metalenses (Zhao et al., 2016a) and metasurfaces (Chen et al., 2018). The second category allows for sub-diffraction focusing without the need of evanescent waves and includes time reversal method (Rupin et al., 2015) and super-oscillations (Shen et al., 2019). Those lenses are generally based on phononic crystals

\footnotetext{
${ }^{*}$ Corresponding author (Email address: zhongk@tongji.edu.cn)
} 
(PCs) (Kushwaha et al., 1993), metamaterials (Liu et al., 2000; Zhu et al., 2018), or metasurfaces (Chen et al., 2011; Cao et al., 2020) to take advantage of their tremendous possibilities of dispersion and phases. The planar gradient-index (GRIN) lenses (Lin et al., 2009; Wu et al., 2011) are of particular interest for focusing, collimating, and energy harvesting (Tol et al., 2016). Usually, the displacement field at the focus is only 3 4 times that of the incident wave (Cui et al., 2019; Liu et al., 2019) which suffers from a severe problem, namely a relatively low signal to noise $(\mathrm{S} / \mathrm{N})$ ratio.

Despite their potential applications such as particle manipulation (Xia et al., 2017) or resonator excitation (Benchabane et al., 2017; Raguin et al., 2019), GRIN lenses encounter a construction dilemma to get the broadband focal spot with FWHM less than half a minimum wavelength inside the lens (labelled as $\lambda_{0}$ hereafter). For example, one possible way to overcome the diffraction limit is to increase the refractive index along the middle of the GRIN lens. Using locally resonant metamaterials to create a large refractive index, either for acoustic (Liu et al., 2019) or for elastic waves (Zhao et al., 2016a), shifts the relevant branches down to low frequency region. This is usually accompanied by strong dispersion so that the effective refractive index may be significantly affected, even by a slight imperfection of the sample (Cui et al., 2019; Liu et al., 2019). So far, FWHM of $~ 0.41$ times the minimum wavelength $\lambda_{0}$ has been reported in an acoustic metamaterial-based GRIN lens (Liu et al., 2019), when the FWHM is retrieved from the profile of normalized energy density but not from the directly measured acoustic pressure. A similar scenario exists for light focusing inside metamaterials based GRIN optical lenses, featuring FWHM less than half the incident wavelength in the free space, but not the minimum wavelength inside the lens $\lambda_{0}$ (Verslegers et al., 2009; Ma et al., 2011). A comparable result is still lacking for elastic waves in solids, not even to mention the broadband challenge. The space-coiling metamaterials such as the zig-zag (Liang and Li, 2012; Liu et al., 2017), helical (Zhu et al., 2016) and Archimedean spiral structures (Fu et al., 2018; Song et al., 2019), may feature broadband large refractive index but they are cumbersome for internal focusing due to the extremely long paths and the intrinsic energy losses. In any case, it is still puzzling to what extent the increase in the refractive index can reduce the FWHM for the focusing of broadband elastic wave, and provides the possibility to obtain a FWHM less than half of the minimum wavelength $\lambda_{0}$ inside the lens.

The GRIN lens constructed by continuously varying the thickness of the plate (Climente et al., 2014; Zhao et al., 2015), presents the advantage of flexibility to modulate in a large extent the refractive index with broadband and identical profiles for the lowest-order antisymmetric Lamb $\left(\mathrm{A}_{0}\right)$ wave, and constitutes therefore a relevant model to gain insights on inner focusing, beyond the resonant metastructures with narrow operation frequency regime.

In this work, we show both experimentally and theoretically that the large refractive index 
along the central axis of a GRIN lens allows to reduce FWHM at the focus of $\mathrm{A}_{0}$ mode to less than half the smallest wavelength in the beam $\lambda_{0}$. We describe the GRIN lenses fabricated by varying the plate thickness, we evidence the broadband sub-diffraction focusing both experimentally and numerically, and we theoretically highlight the underlying physical mechanism. For comparison, we also designed GRIN lenses made of metamaterials, and report numerically the sub-diffraction focusing within a narrow frequency range. Interestingly, the GRIN lenses based on thickness variation features displacement (resp. energy) 30 40 (resp. 1000) times that of incident wave, and they show outstanding achievements even when the transverse size of the GRIN lens is smaller than one incident wavelength $\lambda_{\mathrm{B}}$. To the best of our knowledge, this is the first experimental observation of broadband sub-diffraction and ultra-high energy density focusing of elastic waves in a GRIN lens.

\section{Experimental and numerical observation of sub-diffraction focusing}

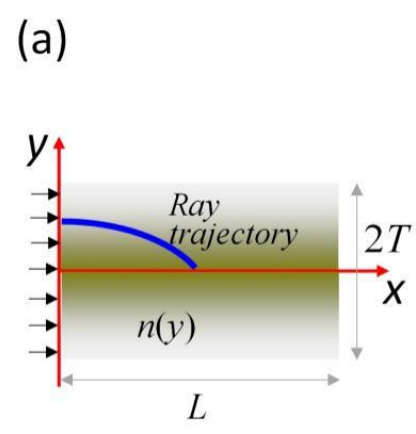

(c)

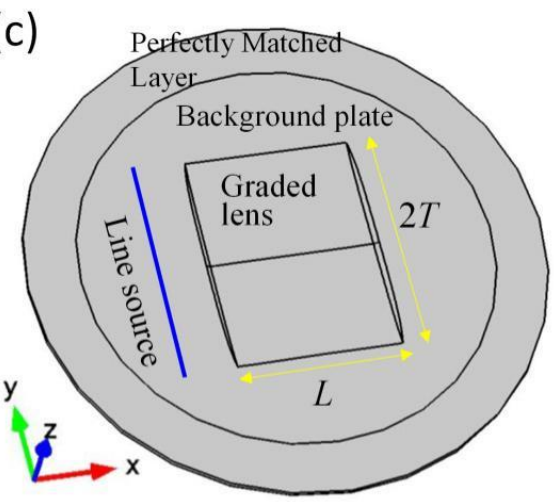

(b)

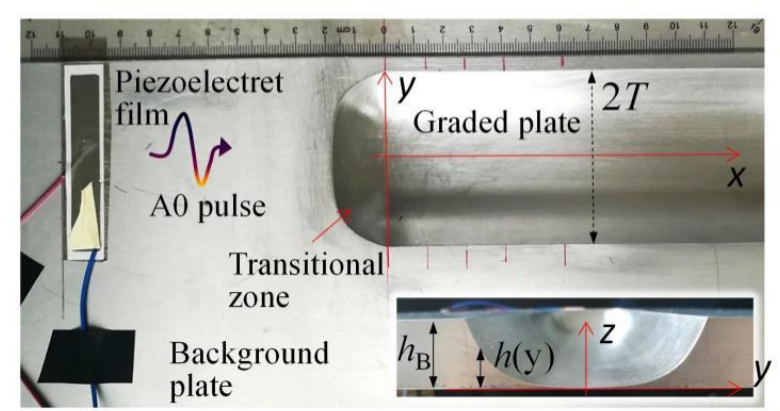

Fig. 1. (a) Scheme of a GRIN lens featuring transversely graded refractive index $n(y)$, (b) top and side (inset) views of the sample of GRIN plate constructed by varying the plate thickness with $n_{0}=6$, and (c) the numerical model to simulate $\mathrm{A} 0$ wave propagation.

Figure 1(a) depicts the general scheme of a GRIN lens, either elastic or optical, featuring the variation of the refractive index $n(y)$ along the transverse direction ( $y$-axis) with dimensions $-T<y<T$ and $0<x<L$. To construct such a GRIN lens on a thin plate, the refractive index $n(y)$ is realized by gradually modulating the plate thickness $h(y)$ along the $y$-axis, as shown in Fig. 1(b). The refractive index $n(y)$ is defined as $n(y)=k(y) / k_{B}$ where $k(y)$ and $k_{\mathrm{B}}$ are the 
wavenumbers inside the lens and from the background plate, respectively. Owing to the dispersion law of $\mathrm{A}_{0}$ mode $k^{4}=\omega^{2} \rho h(y) / D(y)$ (see Section 3) where $D$ is the plate stiffness and $\rho$ the mass density, one can deduce $h(y)=h_{B} / h^{2}(y)$ (Climente et al., 2014) where $h_{\mathrm{B}}$ denotes the thickness of the background plate.

Suppose $n(y)$ follows the hyperbolic secant profile $n(y)=n_{0} \operatorname{sech}(\alpha y)$ where $n_{0}$ is the refractive index along the central axis of the lens and $\alpha$ is the graded coefficient. Such a profile guarantees that all the normally incident waves converge to the same point at the focal distance $f_{l}=\pi / 2 \alpha$ (Gomez-reino et al., 2000; Lin et al., 2009). The parameters $n_{0}$, $\alpha$, and $T$ being related together by $n_{0} \operatorname{sech}(\alpha T)=1$, the thickness varies along the $y$-axis according to $h(y)=h_{B} / n_{0}^{2} \operatorname{sech}^{2}(\alpha y)$; thus, $n(y)$ [resp. $h(y)$ ] decreases (resp. increases) from $n_{0}$ (resp. $h_{0}=h_{B} / h_{0}^{2}$ ) on the middle layer to 1 (resp. $h_{\mathrm{B}}$ ) at the edges of the lens $y= \pm T$.

For typical frequencies of a few tens of $\mathrm{kHz}$, macroscopic GRIN lenses can be manufactured by using a numerically-controlled milling machine. Figure 1(b) shows the top and side views (inset) of a sample made on a 6061-T6 aluminum plate with $n_{0}=6, h_{\mathrm{B}}=18.3 \mathrm{~mm}, T=30 \mathrm{~mm}$, and $\alpha=0.0826 \mathrm{~mm}^{-1}$. Pulses of $\mathrm{A}_{0}$ mode were generated through the excitation of 5-cycles sinusoidal signals under $\pm 100 \mathrm{~V}$ (Zhao et al., 2019) applied to a polypropylene piezoelectret (PP) film $(60 \mathrm{~mm} \times 10 \mathrm{~mm})$ fixed to the plate, by a conductive adhesive, at a distance of about $95 \mathrm{~mm}$ in front of the GRIN lens. The PP film features a typical thickness $0.083 \mathrm{~mm}$ and quasi-static piezoelectric coefficient $d_{33}$ of $\sim 218 \mathrm{pC} / \mathrm{N}$. We used a laser Doppler vibrometer (Polytec vibrometer OFV 2570) to probe the out-of-plane displacement $w$ both inside and outside the GRIN lens, on the plane surface in the back side of the plate (Zhao et al., 2019).

The propagation of the waves was simulated by finite element method (FEM) based on COMSOL Multiphysics, primarily in the time domain by a model similar to the experimental setup in Fig. 1(b) or in the frequency domain by the model depicted in Fig. 1(c). Note that in Fig 1(c), the A0 wave is generated by a line source in front of the graded lens and perfectly matched layers (PMLs) are attached to the background plate to prevent reflections from the boundaries. Further, in both Figs. 1(b) and 1(c), a transition zone (or coupler) is introduced at the inlet of GRIN lens, so as to prevent significant interface reflection, hence disturbing the internal focusing as little as possible. Regarding the material parameters of aluminum, we used the Young's modulus $67.7 \mathrm{GPa}$, Possion's ratio 0.35 and mass density $2700 \mathrm{~kg} / \mathrm{m}^{3}$ (Zhao et al., 2019).

\subsection{Wave focusing characteristics versus $n_{0}$}

We show in Fig. 2(a) the out-of-plane displacement field $|w|$, normalized to the amplitude of the incident wave, inside a lens with $n_{0}=6.00$ at $50 \mathrm{kHz}$. A focal spot centered at $x=16 \mathrm{~mm}$ and $y=0$ is clearly observable. Figure 2(b) presents both the experimental (blue line) and 
numerical (red line) variation of $|w|$ (a.u.) along $y$ axis at $x=16 \mathrm{~mm}$. Of primary interest is the central peak that yields a FWHM of $0.41 \lambda_{0}$ in experiment, in very good agreement with its numerical counterpart of $0.43 \lambda_{0}$, both being less than half the minimum wavelength $\lambda_{0}(9.93$ $\mathrm{mm}$ at $50 \mathrm{kHz}$ ) along the central axis of the structure. When compared with the incident wavelength in the background plate (labelled as $\lambda_{\mathrm{B}}$ hereafter) at $50 \mathrm{kHz}$, i.e. $\lambda_{\mathrm{B}}=45.7 \mathrm{~mm}$, the FWHM is about $\lambda_{\mathrm{B}} / 11.1$. In the experimental results in Fig. 2(b), two sidebands are also observed at the positions expected from the numerical simulations.

We also fabricated two other samples with $n_{0}=2.79$ and 2.03, respectively, and the same width $2 T=60 \mathrm{~mm}$, on a $8 \mathrm{~mm}$ thick plate. We adopted the previous experimental and numerical methods and investigated the focusing inside these lenses of $\mathrm{A}_{0}$ mode at $50 \mathrm{kHz}$. As shown in Fig. 2(c), when $n_{0}=2.79$, the experimental (blue line) and numerical (red line) profiles of $|w|$ (a.u.) along $y$ axis feature $\mathrm{FWHM}=0.47 \lambda_{0}$ and $0.48 \lambda_{0}\left(\lambda_{0}=14.06 \mathrm{~mm}\right)$, respectively, at the focus. Similarly, in Fig. 2(d), we show the case of $n_{0}=2.03$ where FWHM $=0.59 \lambda_{0}$ and $0.62 \lambda_{0}\left(\lambda_{0}=19.15 \mathrm{~mm}\right)$. In both cases, the experimental and numerical results well agree with each other.

(a)

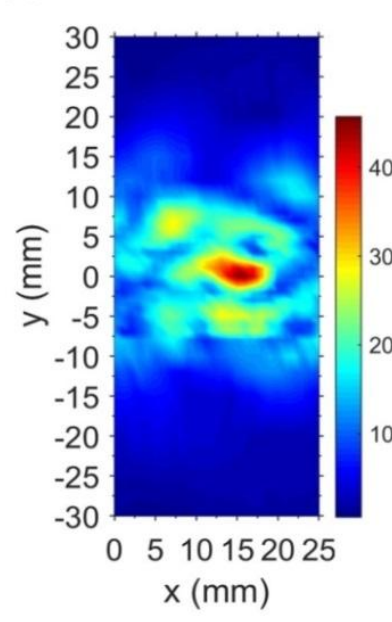

(b)

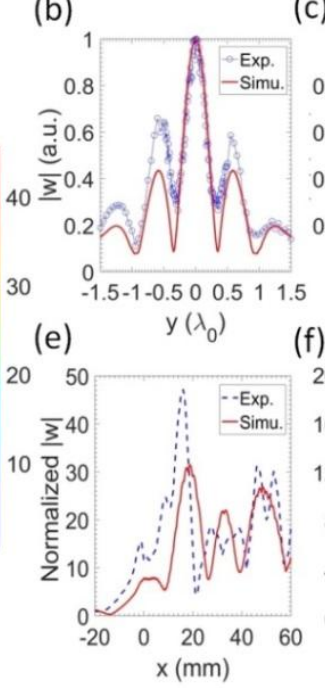

(c)
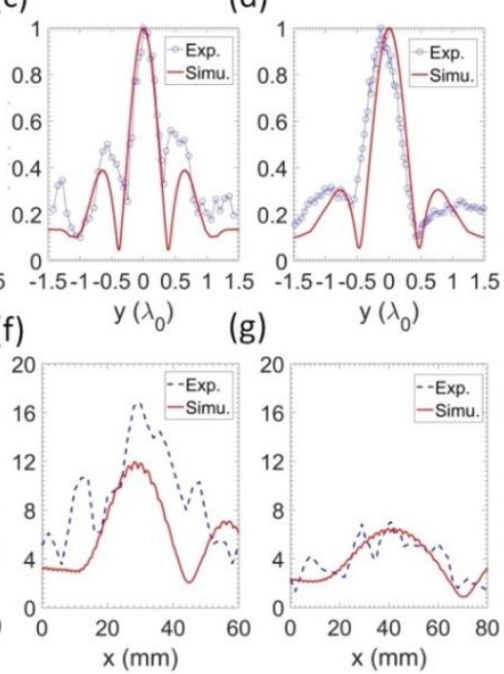

Fig. 2. (a) Experimental mapping of $|w|$ normalized to the amplitude of incident wave inside the lens when $n_{0}=6.0$ at $50 \mathrm{kHz}$. (b)-(d) Experimental (blue line) and numerical (red line) profiles of $|w|$ (a.u.) along the transverse direction at the focal position when $n_{0}$ equates (b) 6.00, (c) 2.79 and (d) 2.03. (e)-(f) Experimental (blue line) and numerical (red line) profiles of $|w|$ normalized to the amplitude of incident wave along the central axis in correspondence with panels (b)-(d), respectively. Note that the experimental profile is achieved at $y=-2 \mathrm{~mm}$ in panel (g) and $y=0$ in panels (e) and (f)

To fully characterize the focus area, we show in Figs. 2(e)-2(g) the experimental (blue line) and numerical (red line) profiles of normalized $|w|$ along the $x$-axis, at position $y=0$. The experimental focal position appears at $x=16,30$ and $41 \mathrm{~mm}$, respectively, to be compared with the numerical values of $x=19.1,28.5$ and $42.1 \mathrm{~mm}$. The evolution of the focal distance 
is summarized in Fig. 9 in SI.

Whatever $n_{0}$, the experimental value $|w|_{\max }$ is larger than its numerical counterpart, which must be attributed to the experimental underestimation of the amplitude of incident wave because of the much lower S/N ratio ( 1.6) than the one [e.g. 16.7 in Fig. 2(e)] at focal position. In addition, Figs. 2(e)-2(g) show the noteworthy characteristic that $|w|_{\max }$ increases all the more as $n_{0}$ becomes larger.

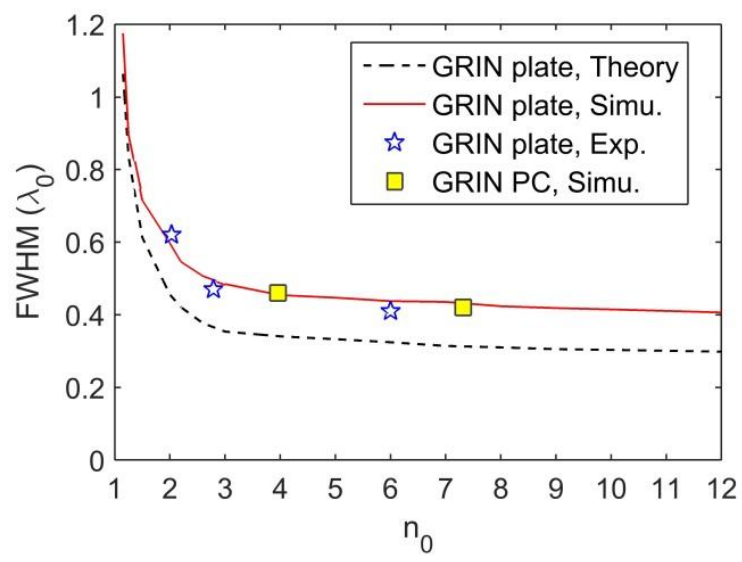

Fig. 3. Theoretical (black line, discussed in Section 3) and numerical (red line) FWHM of the primary transverse peak of $|w|$ at the focal position as a function of $n_{0}$ with $T=30 \mathrm{~mm}$ and $h_{\mathrm{B}}=18.3 \mathrm{~mm}$, at 50 kHz. Stars correspond to experimental results on GRIN plate with $n_{0}=2.03,2.79$ and $6, h_{\mathrm{B}}=8,8$ and $18.3 \mathrm{~mm}$, respectively, at $50 \mathrm{kHz}$ with the same $T=30 \mathrm{~mm}$. Yellow squares correspond to numerical results on GRIN PC plates discussed in section 4 (see also Figs. 12 and 13) with $n_{0}=3.92,7.32, f=3.2$, $12.25 \mathrm{kHz}, T=36.1,62.5 \mathrm{~mm}$ in this order.

To illustrate the sub-diffraction focusing based on GRIN lens, Fig. 3 summarizes the FWHM measured experimentally for different values of $n_{0}$ by blue star symbols: FWHM is less than half a $\lambda_{0}$ for $n_{0}>2.8$. This is further confirmed by the numerical simulations shown as a red line in Fig. 3 where we compute FWHM as a function of $n_{0}$ when $h_{\mathrm{B}}$ and $T$ are fixed to $h_{\mathrm{B}}=$ $18.3 \mathrm{~mm}$ and $T=30 \mathrm{~mm}$, respectively: FWHM progressively decreases to $\sim 0.4 \lambda_{0}$ as $n_{0}$ gradually increases to 12 , evidencing that a high value of $n_{0}$ is essential to reduce the FWHM below half the wavelength $\lambda_{0}$. Moreover, both the experimental and numerical results show that $n_{0}=2.8$ is a threshold for reaching the limit FWHM $<0.5 \lambda_{0}$. Figure 3 can be transposed to Fig. 10 (in SI) when the FWHM is represented in units of $\lambda_{\mathrm{B}}$ instead of $\lambda_{0}$.

\subsection{Wave focusing over a broad frequency range}

To demonstrate the broadband achievement of the proposed lenses, we consider again the experimental sample described by Fig. 1(b) with $n_{0}=6.00$ and we set the central frequency of 5 -cycles excitation signals to $f=39$ and $30 \mathrm{kHz}$ successively. The choice of the 5-cylces excitation signals allows us sweeping the central frequency with a relatively narrow frequency width of $\sim 7 \mathrm{kHz}$. As such, the possible overlapping of different frequencies at the 
same position is avoided, so that the FWHM for a target frequency is characterized with less perturbation. This is significant since the FWHM is of particular interest here, while the broadband generation by using one wave source will be our goal in a future work.

In both cases, the experimental (blue line) profile along $y$-axis of $|w|$ (a.u.) gives rise to a FWHM of $0.44 \lambda_{0}$ at the focal position $x=17 \mathrm{~mm}$ and $18 \mathrm{~mm}$, in order. This is shown in Fig. 4(a) $\left(\lambda_{0}=11.27 \mathrm{~mm}\right.$ at $\left.39 \mathrm{kHz}\right)$ and Fig. $4\left(\right.$ b) $\left(\lambda_{0}=12.86 \mathrm{~mm}\right.$ at $\left.30 \mathrm{kHz}\right)$, in good agreement with the FWHM of $0.43 \lambda_{0}$ derived from the numerical profiles (red lines). We also present in Figs. 4(c)-4(d) the experimental (blue lines) and numerical (red lines) profiles of normalized $|w|$ along the central $x$-axis in correspondence with Figs. 4(a)-4(b), respectively. Along $x$-axis, the experimental and numerical profiles present similar trends for all the considered frequencies.
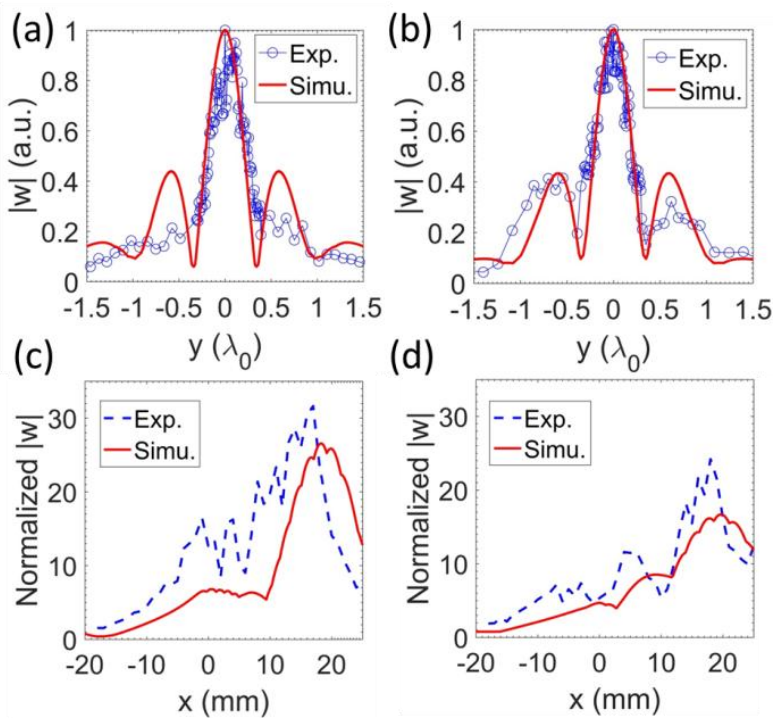

Fig. 4. (a)-(b) Experimental (blue line) and numerical (red line) profiles of $|w|$ (a.u.) along the transverse direction at the focal position with $n_{0}=6.0$ at (a) $39 \mathrm{kHz}$ and (b) $30 \mathrm{kHz}$. (c)-(d) Profiles of normalized $|w|$ along the central axis, in correspondence with (c)-(d). 

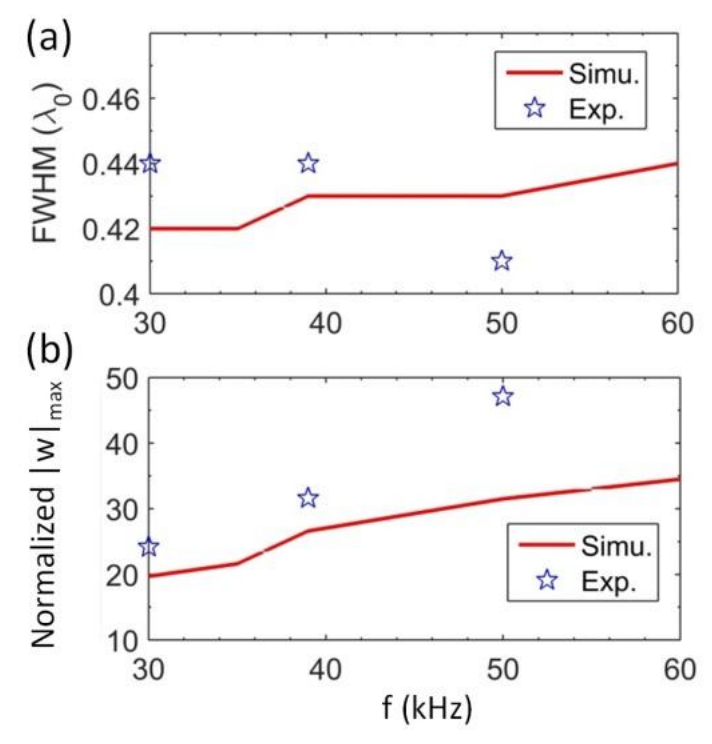

Fig. 5. (a) FWHMs and (b) normalized $|w|_{\max }$ at the focal position normalized to the amplitude of the incident wave as a function of frequency $f$, derived from the numerical (red line) and experimental (stars) results for a GRIN lens featuring $T=30 \mathrm{~mm}, h_{\mathrm{B}}=18.3 \mathrm{~mm}$ and $n_{0}=6.00$.

For the convenience of comparison, the computed results for FWHM between $30 \mathrm{kHz}$ and 60 $\mathrm{kHz}$ are connected to each other by a red line in Fig. 5(a), when the geometrical parameters of the GRIN lens are set to $T=30 \mathrm{~mm}, h_{\mathrm{B}}=18.3 \mathrm{~mm}$, and $n_{0}=6.00$. The measured (stars) FWHMs show good agreement with the values computed at $f=30,39$ and $50 \mathrm{kHz}$. Both groups yield values less than half $\lambda_{0}$. A slight increasing trend is observed for the numerical FWHMs, possibly due to variations of the refractive index as $f$ increases.

Figure 5(b) shows the calculated (red line) and measured (stars) values of $|w|_{\max }$ at the focal position, normalized to the amplitude of incident wave. Both clearly show an increasing trend of $|w|_{\max }$ as $f$ increases. Interestingly, the $|w|_{\max }$ can reach values as high as $\sim 31$ and $\sim 47$ in both simulation and experiment with respect to the incident wave amplitude, whereas this ratio is only about 3 4 in literatures (Wu et al., 2011; Tol et al., 2016; Cui et al., 2019). Further, the energy intensity is proportional to $|w|^{2}$, so that at the focus the energy intensity is about 1000 times that of the incident wave, being about two-order of magnitude higher than known results ( 10) (Liu et al., 2019) obtained from GRIN lens based on metastructures or PCs. It is noteworthy that the ultra-strong energy concentration, combined with FWHM less than half a $\lambda_{0}$, allows us to get the sub-diffraction and ultra-high energy density focusing.

\subsection{Wave focusing versus the transverse size of lens}




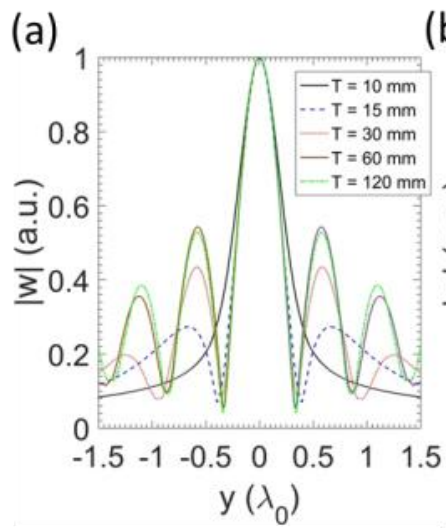

(b)

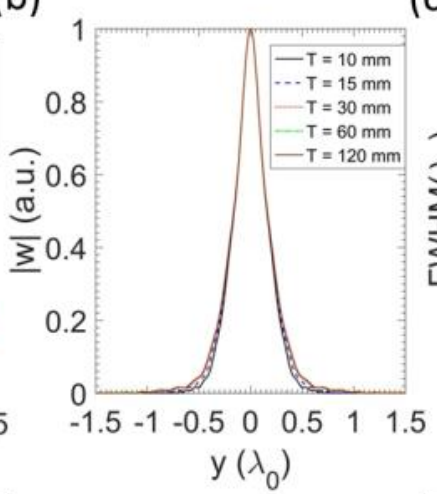

(c)

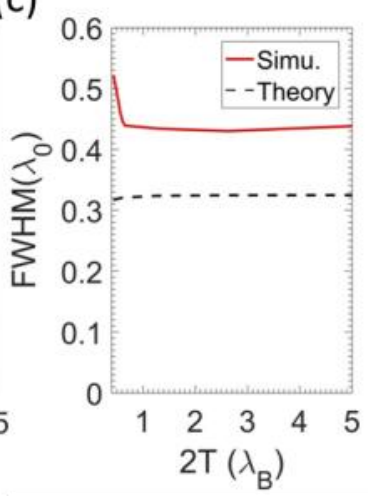

Fig. 6. (a) Numerical and (b) theoretical transverse profiles of $|w|$ (a.u.) at the focal position of GRIN plate lens with $n_{0}=6$ and $h_{\mathrm{B}}=18.3 \mathrm{~mm}$, at $50 \mathrm{kHz}$ for different values of $T$. (c) FWHM in both simulation (red line) and theory (black line) derived from 6(a) and 6(b).

In the following, we investigate the influence of another geometrical parameter in a practical device, i.e. the transverse size of the lens. We show in Fig. 6(a) the computed profiles of $|w|$ along the $y$-axis at the focal position inside the GRIN lens with $n_{0}=6$ and $h_{\mathrm{B}}=18.3 \mathrm{~mm}$ at 50 $\mathrm{kHz}$, for different values of $T$ between $10 \mathrm{~mm}$ and $120 \mathrm{~mm}$. Clearly, the main peak keeps almost the same shape as long as $T$ is larger than $\sim 15 \mathrm{~mm}$ when the transverse size of the lens $2 T$ is about $0.66 \lambda_{\mathrm{B}}$ with the $\mathrm{FWHM}=0.44 \lambda_{0}$.

By using the theoretical model in Section 3, Fig. 6(b) presents the theoretical profiles of $|w|$ along the $y$-axis at focal distance which do not depend on $T$. Figure 6(c) shows the FWHM derived from both numerical (red line) and theoretical (black line) analysis. Both profiles keep a constant value less than $0.5 \lambda_{0}$, until the transverse size of the lens $(2 T)$ becomes a little smaller than one incident wavelength in the background plate $\lambda_{\mathrm{B}}$, making it possible to get sub-diffraction focusing in subwavelength size GRIN lens. The difference between the numerical and theoretical profiles of $|w|$ and the values of FWHM in Fig. 6(c) will be discussed in Section 3 together with theoretical formula.

Figure 6(a) is also of significance to the usual phenomenon relating to sub-diffraction focusing: the FWHM is reduced at the expense of increasing lateral lobes, for either acoustic (Dubois et al., 2015; Zhao et al., 2016a; Shen et al., 2019) or optic waves (Kozawa et al., 2018). From Fig. 6(a), the second lobes are gradually reduced when $T$ decreases while the FWHM of the primary peak is very few affected until $T=15 \mathrm{~mm}$. This provides a different approach to consider this question.

\section{Theoretical model and origin of sub-diffraction focusing}

In this section, we give details on the analytical model used to calculate the distribution of $|w|$ along the $y$-axis at the focal position, and we shed light on the origin of sub-diffraction focusing. We also analyze the influences of another factor in practice, i.e. the transverse size 
of the GRIN lens.

\subsection{Theoretical model}

Let us return to the GRIN lens based on thickness variation in Fig. 1(b), and write the out-of-plane component of the displacement field in the time harmonic form $w(x, y, t)=W(x, y) \omega(i \omega t)$. When the thickness $h$ of the plate is small as compared to the wavelength, the governing equation for the mode $\mathrm{A}_{0}$ can be written as (Climente et al., 2014):

$$
\nabla^{4} W(x, y)-k^{4} W(x, y)=0,
$$

where the wavenumber $k$ satisfies $k^{4}=\omega^{2} \rho h(y) / D(y)$ with the plate stiffness $D(y)=E h^{3}(y) / 12\left(1-v^{2}\right)$, and $v$ is the Poisson's ratio. Equation (1) can be rewritten as

$$
\left(\nabla^{2}+k^{2}\right)\left(\nabla^{2}-k^{2}\right) W(x, y)=0
$$

whose general solution is given by the sum of the solutions of the equations $\nabla^{2} W_{1}+k^{2} W_{1}=0$ and $\nabla^{2} W_{2}-k^{2} W_{2}=0$, i.e. $W=W_{1}+W_{2}$. In general, the solutions of $W_{1}$ stand for the propagative components of the wave, whereas the solutions of $W_{2}$ correspond to the evanescent components. Let us assume that, whatever the coordinate $y,|k(y)|<\left|k_{0}\right|$ with $k_{0}$ denoting the wavenumber along the central axis of the lens; then, the evanescent waves are identically null and Eq. (2) becomes identical to:

$$
\nabla^{2} W_{1}+k^{2} W_{1}=0 .
$$

We show in SI by Fig. 11 that this hypothesis is indeed well verified based on our numerical calculations. We then have $W=W_{1}$ and $k= \pm \sqrt{\omega} \sqrt[4]{\rho h(y) / D(y)}$. The phase velocity becomes $c(y)=\omega / k=\sqrt{\omega h(y)} \sqrt[4]{E / 12 \rho\left(1-v^{2}\right)}$. In analogy to optical lens, the refractive index of the plate lens can be defined by $n(y)=k(y) / k_{B}$ (Zhao et al., 2016a) or $n(y)=c_{B} / c(y)$ where $c_{B}$ is the phase velocity in the background plate. Therefore, the refractive index can be written as $n(y)=\sqrt{h_{B} / h(y)}$, and the thickness of the plate inside the lens is given by $h(y)=h_{B} / n^{2}(y)$ (Climente et al., 2014), where the refractive index satisfies the hyperbolic secant profile $n(y)=n_{0} \operatorname{sech}(\alpha y)$ (Lin et al., 2009), with the focal distance $f_{l}=\pi / 2 \alpha$. If we further remind that $n_{0}, \alpha$ and $T$ satisfy the relationship $n_{0} \times \operatorname{sech}(\alpha T)=1, n(y)$ decreases from $n_{0}$ on the central axis to 1 at the lateral sides $y= \pm T$. As a result, the plate thickness satisfies $h(y)=h_{B} / n_{0}^{2} \operatorname{sech}^{2}(\alpha y)$ within the region $|y| \leq T$ and $h(y)=h_{B}$ if $|y|>T$.

Since Eq. (3) is in the same form as the Helmholtz equation for optical waves in the GRIN fibers, we can use the same method as for optical waves based on geometrical optics to calculate $W_{1}$ using (Gomez-reino et al., 2000):

$$
W_{1}(y, x)=\int_{-T}^{T} K\left(y, y_{0} ; x\right) W_{0}\left(y_{0}\right) d y_{0} \quad(-T<y<T)
$$


In Eq. (4), $W_{0}\left(y_{0}\right)$ represents the field distribution along the local transverse axis $y_{0}$ at position $x=0$, and $K\left(y, y_{0} ; x\right)$ is the propagator function that can be derived by using the stationary phase method (Gomez-reino et al., 2000)

$$
\begin{aligned}
K\left(y, y_{0} ; x\right)= & \left(\frac{V \alpha}{i 2 \pi H_{a}(x)}\right)^{1 / 2} \exp (i V \alpha x) \times \\
& \exp \left\{\begin{array}{l}
\left.i \frac{V}{2 \alpha H_{a}(x)}\left[\begin{array}{l}
\sinh ^{2}(\alpha y) \cos (\alpha x) \\
+\sinh ^{2}\left(\alpha y_{0}\right) \cos (\alpha x)-2 \sinh (\alpha y) \sinh \left(\alpha y_{0}\right)
\end{array}\right]\right\}
\end{array}\right\}^{(-T<y<T)}
\end{aligned}
$$

with $V=k_{0} / \alpha$ and $H_{a}(x)=\sin (\alpha x) / \alpha$. Then, $W_{1}$ at the focal position $x=f_{l}$ is obtained as

$$
\begin{aligned}
W_{1}\left(y, x=f_{l}\right)= & \left(\frac{\alpha k_{0}}{i 2 \pi}\right)^{1 / 2} \exp \left(i V \alpha f_{l}\right) \quad(-T<y<T) . \\
& \times \int_{-T}^{T} \exp \left\{-i \frac{k_{0}}{\alpha} \sinh (\alpha y) \sinh \left(\alpha y_{0}\right)\right\} W_{0}\left(y_{0}\right) d y_{0}
\end{aligned}
$$

Before using Eq. (6) to analyze the distribution of $W_{1}$ along $y$-axis at the focal position, one must determine $W_{0}\left(y_{0}\right)$. For this purpose, we adopt a semi-analytical approach, different from the analytical formula for optical GRIN fibers (Gomez-reino et al., 2000).

Figure 7(a) represents the model where $\mathrm{A}_{0}$ Lamb wave is launched from a background plate $(n=1)$ through a transitional zone to a plate $\left(n=n_{\text {eff }}\right)$ with thickness $h_{B} / n_{\text {eff }}^{2}$. Periodic boundary conditions are applied to the lateral surfaces and perfect matching layers (PMLs) are added on the ends. The calculated $|w|$ on the thinner part, as shown by the red line in Fig. 7(b) when $h_{\mathrm{B}}=18.3 \mathrm{~mm}$ at $50 \mathrm{kHz}$, features always a larger value than the amplitude of the incident wave (Pelat et al., 2020), and highlights an increasing trend in $|w|$ as $n_{\text {eff }}$ increases. In Fig. 7(b), we also show as star symbols the experimental normalized values of $|w|$ at $x=0$ and $y=0$ for our three samples where $n_{0}=6.00,2.79$, and 2.03, respectively. They show good agreement with the calculated values (red line), although the thickness of the plate $h_{\mathrm{B}}$ is different in each sample. 

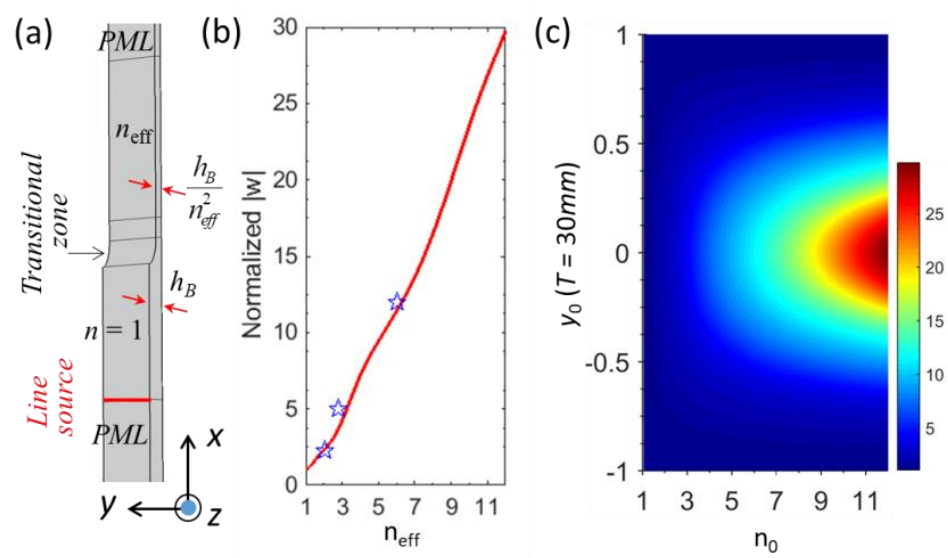

Fig. 7. (a) Scheme of wave propagation from a low-index plate to a high-index one, (b) the amplitude (red line) of $w$ in the high-index plate versus that of the incident wave when $f=50 \mathrm{kHz}$ and $h_{\mathrm{B}}=18.3$ $\mathrm{mm}$, (c) the retrieved value of $W_{0}\left(y_{0}\right)$ versus the amplitude of the incident wave when $f=50 \mathrm{kHz}$ and $T$ $=30 \mathrm{~mm}$. In Fig. 7(b), star symbols are for the experimental values of $|w|$ at $x=0$ and $y=0$ (whereby $n_{\text {eff }}=n_{0}$ ) retrieved from the lens samples with $n_{0}=2.03,2.79$ and 6.00 at $f=50 \mathrm{kHz}$ (the values of $h_{\mathrm{B}}$ are different in each sample).

When the GRIN lens has the hyperbolic secant profile $n(y)=n_{0} \operatorname{sech}(\alpha y)$ for given values of $T$ ( $T=30 \mathrm{~mm}$ here), we can determine the refractive index at any position $y_{0}$ at the entrance of lens by $n\left(y_{0}\right)=n_{0} \operatorname{sech}\left(\alpha y_{0}\right)$; then we retrieve the $W_{0}\left(y_{0}\right)$ from the calculated results shown in Fig. 7(b) by finding the normalized $|w|$ at $n_{\text {eff }}=n\left(y_{0}\right)\left(n_{\text {eff }}<12\right.$ here) at $50 \mathrm{kHz}$. By repeating the procedure, we can get the profile of $W_{0}\left(y_{0}\right)$ at a selected $n_{0}$. Figure 7(c) shows the mapped distribution of $W_{0}\left(y_{0}\right)$ versus $n_{0}$, underlying a decreasing trend from the central axis to the lateral sides of the GRIN lens.

Once $W_{0}\left(y_{0}\right)$ is fixed, we can use Eq. (6) to calculate the distribution of $\left|W_{1}\right|$ (or $\left.|w|\right)$ along the $y$-axis at the focal distance [see for instance Fig. 6(b)], and retrieve the theoretical values of FWHM as a function of $n_{0}$. The result is displayed as a black line in Fig. 3. Obviously, the FWHM continuously decreases to $0.3 \lambda_{0}$ as $n_{0}$ gradually increases to 12 . It should be noted, however, that the theoretical values of FWHM, as shown in Fig. 3 and Fig. 6(c), are smaller than the numerically calculated ones. This difference must be attributed to the following reasons: first, in the derivation of the integral Eq. (6), many simplifications have been used, including the neglect of rotational inertia and shear deformation to get Eq. (1) (Lee and Jeon, 2019). Secondly, when $n_{0}$ takes quite large values, e.g. $n_{0}=6$ in Fig. 6(a), the calculated transverse profiles displays large secondary peaks on either sides of the main peak; however, these sidebands are missing in the theoretical results obtained from the integration formula [see Fig. 6(b)]. This is because in geometrical acoustics (Krylov and Tilman, 2004) (or optics) (Gomez-reino et al., 2000), the $W_{1}$ (or equally $w$ ) can be decoupled into a set of asymptotic series in relation to the wavenumber $k_{\mathrm{B}}$. However, only the first term of these asymptotic series is considered to get the propagator function given in Eq. (5). This truly loses some precision for the analytical solution when $n_{0}$ is large but still presents the evolution trends of 
the central peak versus the $n_{0}$ and/or $T$ in an efficient and adequate way.

Nevertheless, Fig. 3 shows that the theoretical, numerical and experimental results of FWHM decrease simultaneously and consistently as $n_{0}$ increases. Although no lowest limit is expected for the FWHM, in practice lower resolution than $0.40 \lambda_{0}$ will require very high values of $n_{0}$ and hence a very thin plate in the mid part of the GIRN lens. For instance, a thickness of 0.127 $\mathrm{mm}$ is required when $n_{0}=12$. This would make it very challenging for the manufacturing of the lens. In practice one cannot expect a resolution lower than $\sim 0.40 \lambda_{0}$ for FWHM.

The calculation of $W_{0}\left(y_{0}\right)$ also helps the understanding of the important phenomenon noted in Section 2, namely at the focal position, $|w|_{\max }$ is about 31 47 larger than incident wave amplitude. This high value of $|w|_{\max }$ originates from the multiplication of two factors: the amplitude increase of $w$ during the transition from a low-index plate to a higher one, e.g. close to 12 when $n_{0}=6$ [see Fig. 7(b)], and the wave focusing mechanism that can leads to a $|w|_{\max }$ about 3 4 as shown in literatures (Wu et al., 2011; Cui et al., 2019).

\subsection{Discussion on the origin of sub-diffraction focusing}

The subwavelength behavior of FWHM can be analyzed in the following way: at the focal distance, the integral in Eq. (6) implies that the transverse profile of $w$ depends on $k_{0} / \alpha$, $\sinh (\alpha y), \sinh \left(\alpha y_{0}\right)$, and $W_{0}\left(y_{0}\right)$. Typically, the hyperbolic sine function sinh is linear when the variable is smaller than one but dramatically increases as the variable becomes larger. Let us confine the analysis to the central peak for which the coordinate $y$ is approximately in the range of the FWHM: $-0.8 \times F W H M<y<0.8 \times F W H M$ as seen from the results in Figs. 2(b)-2(d) and Figs. 4(a)-4(b). For the GRIN lens with $h_{\mathrm{B}}=18.3 \mathrm{~mm}$ and $T=$ $30 \mathrm{~mm}$ at $50 \mathrm{kHz}$, the product $\alpha \times 0.8 F W H M$ changes from 0.66 to 0.03 as $n_{0}$ increases from 1.15 to 12 so that within the central peak the product $\alpha y$ is smaller than one. As a result, we can make the approximation $\sinh (\alpha y)=\alpha y$ within the central peak. Under this condition, the

integration in Eq. (6) translates into $\int_{-T}^{T} \exp \left\{-i k_{0} y \sinh \left(\alpha y_{0}\right)\right\} W_{0}\left(y_{0}\right) d y_{0}$ for the central peak. 

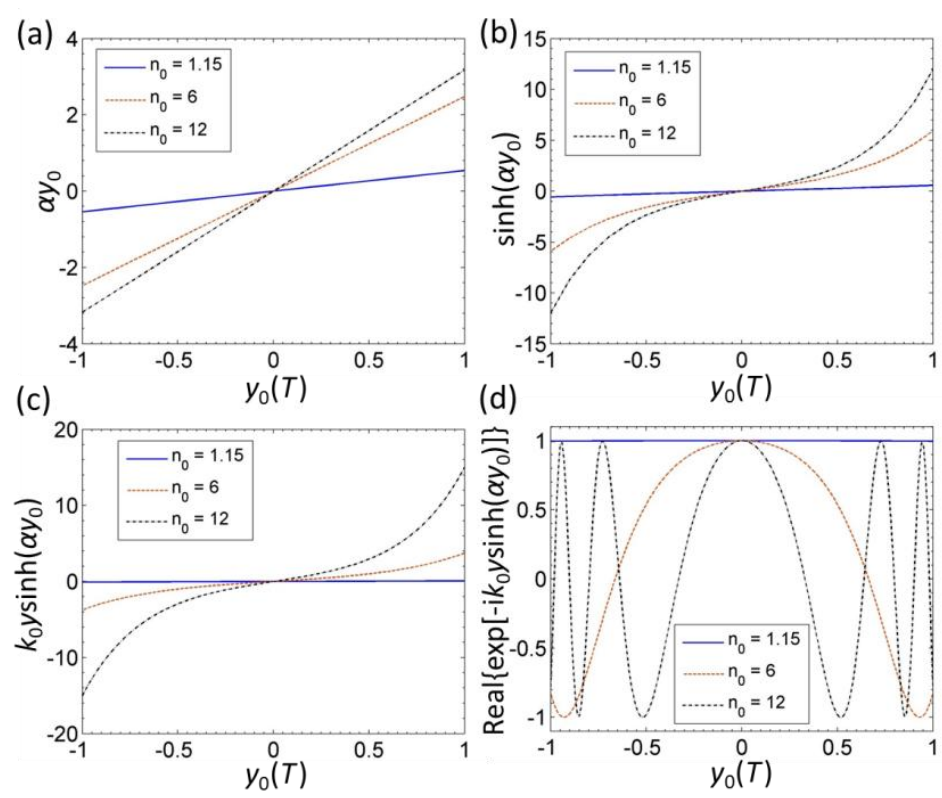

Fig. 8. Calculated profiles of (a) $\alpha y_{0}$ and (b) $\sinh \left(\alpha y_{0}\right)$ for $n_{0}=1.15$ (blue solid line), 6 (red dashed line) and 12 (black point-dashed line) when $f=50 \mathrm{kHz}, h_{\mathrm{B}}=18.3 \mathrm{~mm}$ and $T=30 \mathrm{~mm}$. Related profiles of (c) $k_{0} y \times \sinh \left(\alpha y_{0}\right)$ and (d) the real part of $\exp \left[-i k_{0} y \times \sinh \left(\alpha y_{0}\right)\right]$ both with a given $y=1 \mathrm{~mm}$.

Now let us see the variations of $\sinh \left(\alpha y_{0}\right)$ whereby $\alpha y_{0}$ changes from $-\alpha T$ to $\alpha T$. Remembering that $\alpha T=\operatorname{sech}^{-1}\left(1 / n_{0}\right)$, one can calculate the profiles of $\alpha y_{0}$ with $n_{0}=1.15,6$ and 12, as shown by the blue solid, red dashed and black point-dashed lines respectively in Fig. $8(\mathrm{a})$, when $f=50 \mathrm{kHz}, h_{\mathrm{B}}=18.3 \mathrm{~mm}$ and $T=30 \mathrm{~mm}$. The product $\alpha T$ changes gradually from 0.54 to 3.18 as $n_{0}$ increases from 1.15 to 12 . Therefore, for a large $n_{0}$, for example $n_{0}=$ 12 , the $\alpha y_{0}$ shall change between -3.18 and 3.18, beyond \pm 1 . Accordingly, the calculated $\sinh \left(\alpha y_{0}\right)$ as shown by the black point-dashed line in Fig. 8(b) for a large $n_{0}=12$, can change dramatically as compared to the profile with a small $n_{0}=1.15$ noted by the blue solid line. The big variation range of $\sinh \left(\alpha y_{0}\right)$ with a large $n_{0}$ allows for the rapid oscillation of the exponential term or propagator function $\exp \left\{-i k_{0} y \sinh \left(\alpha y_{0}\right)\right\}$. Indeed, this rapid oscillation is usually achieved by using the evanescent wave (Pendry, 2000; Zhao et al., 2016a) or super-oscillation method (Shen et al., 2019) , as the base of creating sub-diffraction focusing.

To give further evidence, let us set $y=1 \mathrm{~mm}$ which is at the vicinity of the $0.5 \times F W H M$ when $n_{0}=12$. Figure 8 (c) presents the profiles of $-i k_{0} y \sinh \left(\alpha y_{0}\right)$ when $n_{0}=1.15$ (blue solid line), 6 (red dashed line) and 12 (black point-dashed line), which maintains similar profiles as their counterparts in Fig. 8(b), featuring the slightly enlarged amplitude range. After that, Fig. 8 (d) gives the profiles of the real part of the propagator function $\exp \left\{-i k_{0} y \sinh \left(\alpha y_{0}\right)\right\}$. Obviously, the black point-dashed line for $n_{0}=12$ oscillates much more quickly than the almost constant blue solid line for $n_{0}=1.15$. Similar phenomenon can be observed for the 
imaginary part of the $\exp \left\{-i k_{0} y \sinh \left(\alpha y_{0}\right)\right\}$. This fast oscillation of propagator function for the large $n_{0}$ reduces quickly the integral of $\int_{-T}^{T} \exp \left\{-i k_{0} y \sinh \left(\alpha y_{0}\right)\right\} W_{0}\left(y_{0}\right) d y_{0}$, as compared to the corresponding value for a small $n_{0}$. It is noteworthy that, the modulations of $\sinh \left(\alpha y_{0}\right)$ in Fig. 8(b) and $-i k_{0} y \sinh \left(\alpha y_{0}\right)$ in Fig. 8(c) are not linear as $n_{0}$ changes, but occur in an accelerated way. Consequently, the oscillations number in Fig. 8(d) when $n_{0}=12$ (about 4.5) is more than doubled as compared to $n_{0}=6$ (with approximately one oscillation). This gives the possibility of getting even smaller FWHM normalized to the relevant $\lambda_{0}$ when $n_{0}=12$ than the normalized FWHM when $n_{0}=6$.

We can also see that $\alpha T=\operatorname{sech}^{-1}\left(1 / n_{0}\right)$ is independent of $T$, so that changing $T$ cannot disturb the central peak profile in theory [see Fig. 6(b)], while the focal distance $f_{l}=\pi / 2 \alpha$ is modified. The component $W_{0}\left(y_{0}\right)$, however, offsets the effect of rapid oscillation to a certain extend. While the behavior of $W_{0}\left(y_{0}\right)$ does not matter in the integration domain when the exponential $\exp \left\{-i k_{0} y \sinh \left(\alpha y_{0}\right)\right\}$ oscillates fast, it can become significant in the domain where the exponential oscillates slowly. To demonstrate this, we replaced $W_{0}\left(y_{0}\right)$ par 1 in Eqs. (4) and (6) and obtained a FWHM as small as $0.14 \lambda_{0}$ in theory when $n_{0}=6$ that is significantly different from the computed value of $0.41 \lambda_{0}$.

Finally, we further discuss on one hidden factor in Eq. (7). Indeed, when designing the GRIN plate with a given $T$, we first define the value of $n_{0}$ and then calculate the plate thickness $h(y)$ according to $h(y)=h_{B} / n_{0}^{2} \operatorname{sech}^{2}(\alpha y)$. The plate thickness $h_{0}$ in the middle of the layer is given by $h_{0}=h_{B} / n_{0}^{2}$. Then, the wavenumber $k_{0}$ of $\mathrm{A}_{0}$ mode in the middle layer of the lens is calculated at a given frequency (Zhao et al., 2013). Let us call this selected $n_{0}$ as the nominal refractive index. For the sake of clarity, the effective refractive index defined by $k_{0} / k_{B}$ is called the actual refractive index and we refer to it with another symbol $n_{8}$. The $n_{0}$ is almost equal to $n_{0}$ when $n_{0}$ features a small value but not large ones. For example, when $h_{\mathrm{B}}=18.3$ $\mathrm{mm}$ at $f=50 \mathrm{kHz}$, the $\sigma_{Q} q_{Q}\left(k_{0} / k_{B}\right)$ increases gradually from 1.07 to 15.2 as $n_{0}$ ranges from 1.15 to 20 . Due to this discrepancy in Eq. (6), we always used the calculated value of $k_{0}\left(n_{0} k_{B}\right)$ for a given thickness of $h_{0}=h_{B} / n_{0}^{2}$ instead of the value of $n_{0} k_{\mathrm{B}}$ as in the optical GRIN fibers (Gomez-reino et al., 2000). However, the nominal refractive index $n_{0}$ is kept as in $h(y)=h_{B} / n_{0}^{2} \operatorname{sech}^{2}(\alpha y)$ to simplify the engineering of the lens and a good precision in predicting the focal distance.

\section{Numerical Results on GRIN PC lens}

As a matter of comparison, we have numerically studied the wave focusing inside the metamaterial-based GRIN PC lenses consisting of subwavelength pillars and holes (Rupin et al., 2014; Bilal et al., 2017; Colquitt et al., 2017) on a plate. Relevant results are presented in Appendix C. The calculated FWHMs for the GRIN PC lens that features $n_{0}=7.32$ and 3.92, respectively at $3.2 \mathrm{kHz}$ and $12.25 \mathrm{kHz}$, are summarized by the yellow square symbols in Fig. 3. The values of $0.42 \lambda_{0}$ and $0.46 \lambda_{0}$ present a good agreement with the results obtained with 
GRIN plate made by varying plate thickness (red line). However, the pillared metamaterials are strongly dispersive, as evidenced by the flat branches in Fig. 12(b). Therefore, $n_{0}$ changes quickly when $f$ shifts away from the target frequency and the $n(y)$ has no longer a hyperbolic secant profile. Hence, sub-diffraction focusing with the metamaterial based GRIN lens is achieved in a relatively narrow frequency band only. Further, the amplitude of $u_{z}$ at the focusing is also quite small in comparison to the one inside the GRIN plate.

\section{Conclusions}

In summary, we presented the first experimental and theoretical study of broadband sub-diffraction focusing of elastic wave inside GRIN lenses based on thickness modulation. The large refractive index in the middle of the structure is particularly important to reduce the FWHM of inner focusing to less than half the minimum wavelength $\lambda_{0}$. A comprehensive explanation of the physical mechanism is given for this inside sub-diffraction focusing without introducing evanescent waves. Notable features of our design include the broadband frequency range, small transverse size comparable or even less than one incident wavelength in the background plate $\lambda_{\mathrm{B}}$, and an amplitude of displacement (resp. energy) at the focus about 30 40 (resp. 1000) times of that of incident wave. We also designed metamaterials based GRIN lenses, and numerically put into evidence the sub-diffraction focusing within a narrow frequency band. Our results draw a picture on the essential parameters for controlling the FWHM of $A_{0}$ wave in GRIN lenses, without and with metastructures, and pave the way to the design of new structures for focusing of other types of waves or phonons.

Specifically, the broadband sub-diffraction and ultra-high energy density focusing given by our GRIN lenses can be used to enhance the signals or harvest energy (Tol et al., 2016; Liu et al., 2019), considering that the energy density amplification is an essential step for both of them. Typically, the resonators are adopted to enhance the energy density or relevant physical parameters within a narrow frequency. Our broadband GRIN lenses present quite an advantage, as evidenced by the $\sim 1000$ times energy amplification at the focal position, for resolving this problem even in presence of a significant noise in the incident wave. Of practical engineering interest, it is possible to implement one or several GRIN lenses in plate structures to capture the weak signals generated or scattered by small defects, so as to monitor their location reversely. The operation frequency range is wide.

Acknowledgements: This work was supported by the National Natural Science Foundation of China (Grant Nos. 11602174, 11772106, 11872282 and 52027816), and Shanghai Science and Technology Innovation Plan (Grant No. 20ZR1462700). Jinfeng Zhao would like to acknowledge the experimental helps given by $\mathrm{PhD}$ student Chao SONG from Tongji University. 


\section{Supplementary materials}

\section{Appendix A. Evolution of focal distance and FWHM (in $\lambda_{\mathrm{B}}$ ) versus $\boldsymbol{n}_{0}$}

In this section, we give an example of the evolution of focal distance $f_{l}$ of a GRIN lens as a function of $n_{0}$. When $n(y)$ has the hyperbolic secant profile, the focal distance $f_{l}$ is given by $f_{l}$ $=\pi / 2 \alpha$. Let us consider a GRIN lens with $T=30 \mathrm{~mm}, h_{\mathrm{B}}=18.3 \mathrm{~mm}$ and the refractive index relationship $n_{0} \operatorname{sech}(\alpha T)=1$. Figure 9 displays the theoretical (black line) and numerical (red line) results for $f_{l}$ as a function of $n_{0}$ at $50 \mathrm{kHz}$. Both are in good agreement, especially at high $n_{0}$. For comparison, we show also as stars the measured focal distances at $50 \mathrm{kHz}$ for three samples with $n_{0}=6.00,2.79$ and 2.03, respectively. Although the thickness of the plate $h_{\mathrm{B}}$ is different in each sample, the experimental results are consistent with both the theoretical and numerical ones.

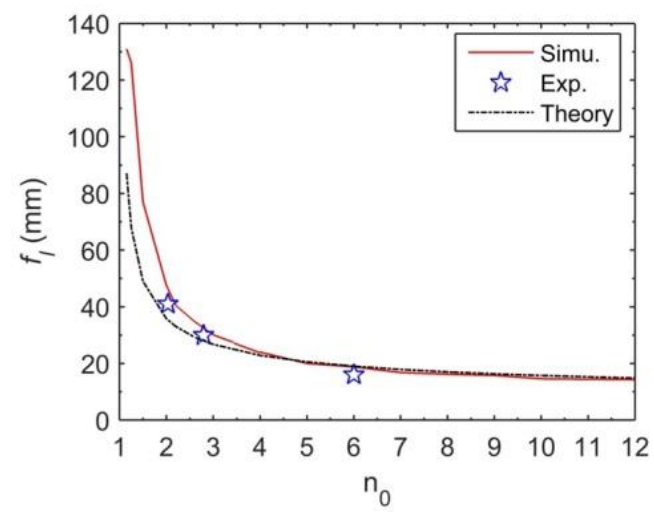

Fig. 9. Numerical (red line), experimental (star symbols), and theoretical profiles (black line) of focal distance of GRIN plate versus $n_{0}$ when $T=30 \mathrm{~mm}$ at $50 \mathrm{kHz}$.

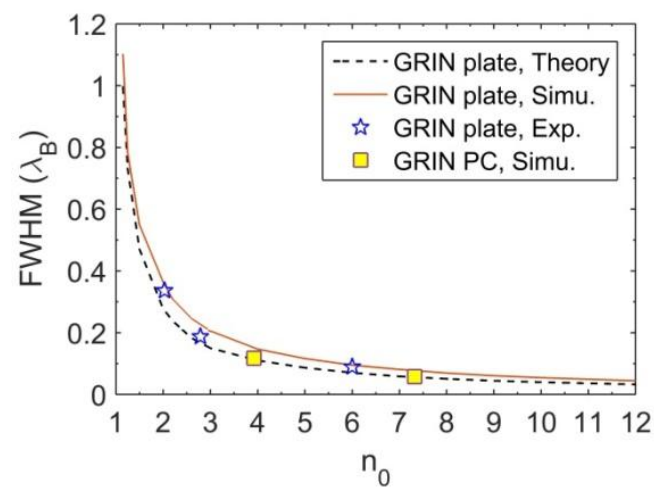

Fig. 10. Theoretical (black line) and numerical (red line) FWHM in units of $\lambda_{\mathrm{B}}$ of the primary transverse peak of $|w|$ at the focal position as a function of $n_{0}$ with $T=30 \mathrm{~mm}$ and $h_{\mathrm{B}}=18.3 \mathrm{~mm}$, at 50 kHz. Experimental results (stars) on GRIN plate correspond to $n_{0}=2.03,2.79$ and 6, with $h_{\mathrm{B}}=8,8$ and $18.3 \mathrm{~mm}$, respectively, at $50 \mathrm{kHz}$ with the same $T=30 \mathrm{~mm}$. Numerical results (squares) on GRIN PC plates (see also Figs. 12 and 13) correspond to $n_{0}=3.92,7.32$, with $f=3.2,12.25 \mathrm{kHz}$ and $T=36.1$, $62.5 \mathrm{~mm}$ in this order. 
For the convenience of demonstrating the performance of GRIN lens based on plate thickness modulation or metamaterials, the FWHM shown in Fig. 3 in units of $\lambda_{0}$ are presented in Fig. 10 in units of $\lambda_{\mathrm{B}}$. From this latter figure, the theoretical (black line) and numerical (red line) FWHMs calculated for the lens with thickness variations agree well with each other; they magnify a decreasing trend as $n_{0}$ increases for the GRIN plate lens with $T=30 \mathrm{~mm}$ and $h_{\mathrm{B}}=$ $18.3 \mathrm{~mm}$, at $50 \mathrm{kHz}$, and the FWHM can be smaller than $0.1 \lambda_{\mathrm{B}}$. On the other hand, the experimental FWHM as noted by the star symbols agree well with the calculated and theoretical profiles of FWHM when $n_{0}=2.03,2.79$ and 6 at $50 \mathrm{kHz}$, even the $h_{\mathrm{B}}$ is different in each case. The computed FWHMs for the GRIN PC labelled by the square markers matches well with those computed for the GRIN plate lens. However, the GRIN PC usually works in narrow frequency range, and it is necessary to adapt for each $n_{0}$ the operation frequency and transverse size of lens; whereas the GRIN plate can perform even well in a broad band and is robust with respect to the transverse size of the plate lens.

\section{Appendix B. Discussion on the influence of evanescent waves}
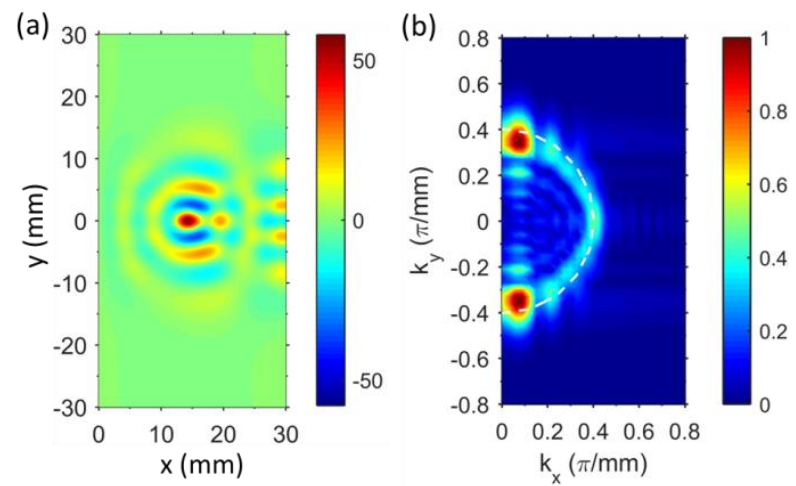

Fig. 11. (a) Snapshot of $w$ normalized to the amplitude of incident wave inside the graded lens with $h_{\mathrm{B}}$ $=18.3 \mathrm{~mm}, T=30 \mathrm{~mm}$, and $n_{0}=12$ at $f=50 \mathrm{kHz}$. (b) Two-dimensional Fourier transform together with the equifrequency contour (white dashed line) at $50 \mathrm{kHz}$ for the middle layer with the thickness 0.127

$\mathrm{mm}$.

In this section we discuss the possible influence of evanescent waves on the focus size. Figure 11(a) displays the amplitude of the out-of-plane component $w$, normalized to the amplitude of the incident wave inside a GRIN lens with $h_{\mathrm{B}}=18.3 \mathrm{~mm}, T=30 \mathrm{~mm}$ and $n_{0}=12$ at $50 \mathrm{kHz}$. The wave is focused at a distance $x=14.3 \mathrm{~mm}$, in good agreement with the theoretical value $f_{l}$ $=14.8 \mathrm{~mm}$. Figure 11(b) shows the normalized two-dimensional Fourier Transform of the spatial distribution of $w$ in Fig. 11(a), together with the equifrequency contour (EFC) of $\mathrm{A}_{0}$ mode at $50 \mathrm{kHz}$, represented by a white dashed line, for the central layer where the thickness is $0.127 \mathrm{~mm}$. The components of the Fourier transformation well overlap with EFC with the wavenumber $k_{0}=0.43 \pi / \mathrm{mm}$. The occurrence of evanescent waves on the middle layer requires $k_{x}^{2}+k_{y}^{2}>k_{0}^{2}$ and consequently large components of Fourier transform beyond the 
EFC (Zhao et al., 2016a). However, this is not observable in Fig. 11(b) and therefore, the evanescent waves do not contribute, or very little, to the inner focusing.

Further, the upper spot $\left(k_{y}>0\right)$ in Fig. 11(b) derives from the converging incident wave from the lower side of GRIN lens $(y<0)$ in Fig. 11(a), for which the $k_{y}$ component tilts gradually from 0 to a quite large value at the focal position (Zhao et al., 2016a). The lower spot $\left(k_{y}<0\right)$ in Fig. 11(b) can be understood in a similar way.

\section{Appendix C. Detailed Numerical Results on GRIN PC lens}
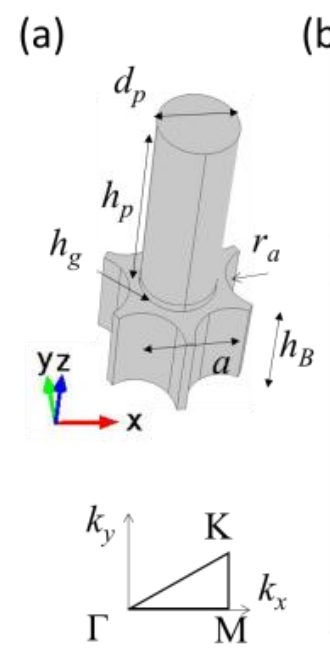

(b)

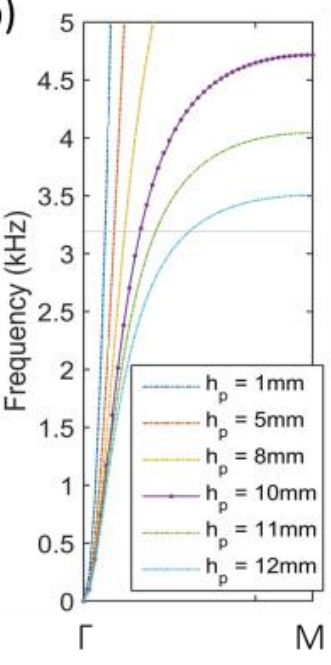

(c)

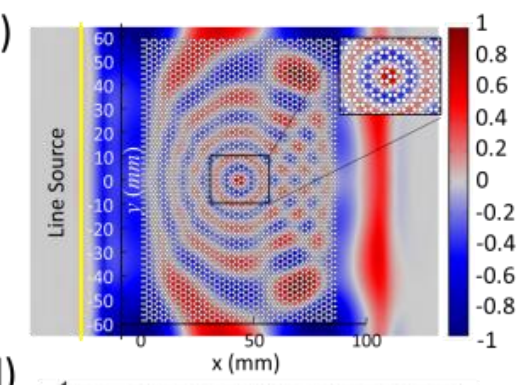

(d)

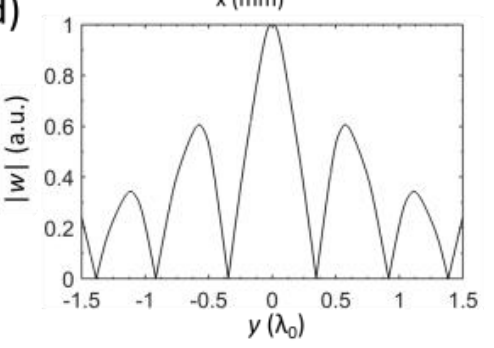

Fig. 12. (a) Schematic view of the unit cell of the GRIN lens made of holes and pillars in a plate, together with its geometrical parameters and the first irreducible Brillouin zone. (b) Dispersion curves of $\mathrm{A}_{0}$ mode versus pillar's height $h_{p}$. (c) Calculated distribution of normalized $w$ at $3.2 \mathrm{kHz}$ and the zoom in of the focal spot (inset). (d) Computed transverse profile of $|w|$ (a.u.) at the focal distance in 12(c).

The GRIN PC lenses are constituted in such a way that an array of steel pillars are fixed by the epoxy adhesive on the center of identical air holes perforated in a 6061-T6 aluminum plate in a honeycomb lattice (Cui et al., 2019). Figure 12(a) depicts the unit cell, the geometrical characteristics, and the first irreducible Brillouin zone of PC plate. Figure 12(b) shows the dispersion of $\mathrm{A}_{0}$ mode, along $Г \mathrm{M}$ direction of a perfect $\mathrm{PC}$ for various heights $h_{p}$ of the pillars, magnifying a decreasing trend as $h_{p}$ increases. The other parameters are (in mm) $a$ $=1.443, r_{a}=0.65, d_{\mathrm{p}}=1.24, h_{\mathrm{B}}=1.5, h_{\mathrm{g}}=0.135$. The Young's modulus, Possion's ratio, and mass density are $199 \mathrm{GPa}, 0.25$ and $7850 \mathrm{~kg} / \mathrm{m}^{3}$ for the steel; $5.1 \mathrm{GPa}, 0.38$, and $1200 \mathrm{~kg} / \mathrm{m}^{3}$ for epoxy resin (Cui et al., 2019). Notice that $\Gamma M$ of GRIN PC is parallel to both the $x$ - and $y$-axes, and the crystallographic anisotropy at any layer is very weak for A0 mode. In this condition, the refractive index is defined by $n=k_{\Gamma M} / k_{\mathrm{B}}$ where $k_{\Gamma M}$ and $k_{\mathrm{B}}$ are the wavenumber along $\Gamma M$ direction in the PC plate and in pure aluminum plate, respectively (Cui et al., 2019). 
The GRIN PC lens is designed by tailoring the graded values of $h_{p}$ such that $n_{0}=7.32, T=$ 43.3a, $\alpha=0.0526 a^{-1}$ and $n(y= \pm T)=1.48$ at $3.2 \mathrm{kHz}$. With these characteristics, the GRIN lens enables for deep subwavelength control of the waves since $a=\lambda_{\mathrm{B}} / 46.6$, with $\lambda_{\mathrm{B}}=67.2$ $\mathrm{mm}$. Figure 12(c) shows the computed distribution of $w$ normalized to the amplitude of the incident wave at $3.2 \mathrm{kHz}$. The incident wave converges to a spot centered at $x=42.5 \mathrm{~mm}$, in very good agreement with the theoretical focal distance expected at $43.1 \mathrm{~mm}$. Figure 12(d) shows the transverse profile of $|w|$ (a.u.) at the focal position, featuring a FWHM of about $0.42 \lambda_{0}\left(\lambda_{0}=9.29 \mathrm{~mm}\right)$ for the central peak that is less than half $\lambda_{0}$.

(a)

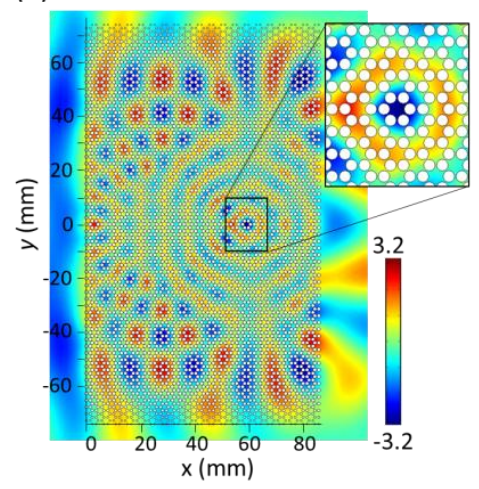

(b)

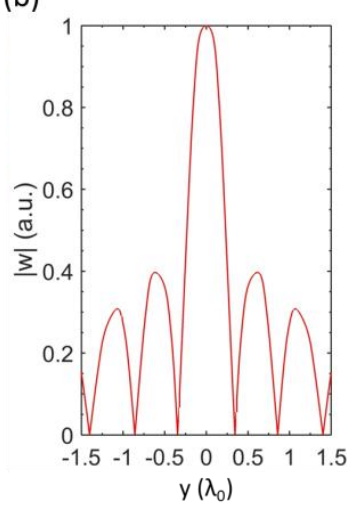

Fig. 13. (a) Calculated distribution of $w$ normalized to the amplitude of the incident wave inside a GRIN PC lens featuring $n_{0}=3.92$ and the zoom in of the focal spot (see inset) when $f=12.25 \mathrm{kHz}$, (b) the transverse profile of $|w|$ (a.u.) at the focal position.

For more details on wave focusing inside the GRIN PC lens with a smaller $n_{0}$, we designed another GRIN PC lens with (in mm) $a=1.443, d_{\mathrm{p}}=1.24, h_{\mathrm{B}}=1.5$, and $h_{\mathrm{g}}=0.135$. The GRIN PC lens is composed of three parts: within $-43.3 a<y<43.3 a$, the pillar height $h_{p}$ changes gradually from $5 \mathrm{~mm}$ to $1 \mathrm{~mm}$ while the radius of the air holes is kept constant to $r_{a}=0.65$ mm; within $-51.1 a<y<-43.3 a$ and $43.3 a<y<51.1 a, r_{a}$ gradually decreases from $0.65 \mathrm{~mm}$ to $0.28 \mathrm{~mm}$ at the most lateral sides while $h_{p}$ is kept constant to $1 \mathrm{~mm}$. As such, the GRIN PC lens features $n_{0}=3.92$ on the middle layer and $n(y= \pm 51.1 a)=1.16$ at the most lateral sides at $12.25 \mathrm{kHz}$. Figure 13(a) shows the calculated distribution of $w$, normalized to the amplitude of the normally incident plane wave when $f=12.25 \mathrm{kHz}$. The focusing of the incident wave towards a spot at $x=59.17 \mathrm{~mm}$ is clearly observable. The transverse profile of $|w|$ (a.u.) measured at the focal position is presented in Fig. 13(b): the FWHM of $0.46 \lambda_{0}\left(\lambda_{0}=8.76 \mathrm{~mm}\right)$ is achieved which is larger than the one $0.42 \lambda_{0}$ for $n_{0}=7.32$, while the second lobes in Fig. 13(b) is smaller than their counterparts in Fig. 12(d). As shown in Fig. 3, the calculated FWHMs that correspond to $n_{0}=7.32$ and 3.92 (yellow square markers) present a good agreement with the results obtained with GRIN plate made by varying plate thickness (red line), even although the operation frequency is different with each model.

However, because of their discretized structure, each horizontal layer in a GRIN lens based on 
metamaterials may not behave exactly as expected. For example, the coupling efficiency between the pillars and the substrate varies along each horizontal layer. On the other hand, the wave vector $\mathbf{k}$ in the middle layer being close to $\mathrm{M}$ or $\mathrm{K}$ point of the Brillouin zone, the assumption of homogenization of the effective refractive index is not strictly respected any more. Based on these points, the accuracy of Eq. (6) may be affected when $n_{0}$ takes large values. The GRIN lenses based on elastic metamaterials must also face another common challenge, i.e. impedance mismatch between the lens and the background plate that can result in strong interface reflection. This reflection highly impacts the amount of energy converging towards the focal spot, as it is the case in both Figs. 13(a) and 12(c) where the amplitudes at the focus are much reduced in comparison to the GRIN lens based on plate thickness variation. To prevent energy losses, a solution would consist of introducing a carefully designed transitional zone in front of the GRIN PC lens, as it is the case in Fig. 1(b).

\section{References}

Benchabane, S., Salut, R., Gaiffe, O., Soumann, V., Addouche, M., Laude, V., Khelif, A., 2017. Surface-Wave Coupling to Single Phononic Subwavelength Resonators. Phys. Rev. Applied 8, 034016. Bilal, O.R., Foehr, A., Daraio, C., 2017. Observation of trampoline phenomena in -printed metamaterial plates. Extreme Mech. Lett. 15, 103-107.

Cao, L., Yang, Z., Xu, Y., Fan, S.-W., Zhu, Y., Chen, Z., Li, Y., Assouar, B., 2020. Flexural wave absorption by lossy gradient elastic metasurface. J. Mech. Phys. Solids 143, 104052.

Chen, J., Xiao, J., Lisevych, D., Shakouri, A., Fan, Z., 2018. Deep-subwavelength control of acoustic waves in an ultra-compact metasurface lens. Nat. Commun. 9, 4920.

Chen, P.-Y., Farhat, M., Guenneau, S., Enoch, S., Alù, A., 2011. Acoustic scattering cancellation via ultrathin pseudo-surface. Appl. Phys. Lett. 99, 191913.

Climente, A., Torrent, D., Sánchez-Dehesa, J., 2014. Gradient index lenses for flexural waves based on thickness variations. Appl. Phys. Lett. 105, 064101.

Colquitt, D.J., Colombi, A., Craster, R.V., Roux, P., Guenneau, S.R.L., 2017. Seismic metasurfaces: Sub-wavelength resonators and Rayleigh wave interaction. J. Mech. Phys. Solids 99, 379-393.

Cui, X., Zhao, J., Boyko, O., Bonello, B., Zhong, Z., 2019. Multi-branch subwavelength focusing of the lowest-order antisymmetric Lamb mode in a gradient-index phononic crystal. Int. J. Mech. Sci. 157-158, 677-683.

Dong, H.-W., Zhao, S.-D., Wang, Y.-S., Zhang, C., 2017. Topology optimization of anisotropic broadband double-negative elastic metamaterials. J. Mech. Phys. Solids 105, 54-80.

Dubois, M., Bossy, E., Enoch, S., Guenneau, S., Lerosey, G., Sebbah, P., 2015. Time-driven superoscillations with negative refraction. Phys. Rev. Lett. 114, 013902.

Fu, Y., Li, J., Xie, Y., Shen, C., Xu, Y., Chen, H., Cummer, S.A., 2018. Compact acoustic retroreflector based on a mirrored Luneburg lens. Phys. Rev. Mater. 2, 105202.

Gomez-reino, C., Perez, M.V., Bao, C., Flores-arias, M.T., Vidal, S., 2000. Diffraction-free and diffraction-limited propagation of light in graded-index planar waveguides with hyperbolic secant refractive index profile. J. Mod. Optic. 47, 91-102.

Kozawa, Y., Matsunaga, D., Sato, S., 2018. Superresolution imaging via superoscillation focusing of a radially polarized beam. Optica $5,86-92$. 
Krylov, V.V., Tilman, F.J.B.S., 2004. Acoustic 'black holes' for flexural waves as effective vibration dampers. J. Sound Vib. 274, 605-619.

Kushwaha, M.S., Halevi, P., Dobrzynski, L., Djafari Rouhani, B., 1993. Acoustic band structure of periodic elastic composites. Phys. Rev. Lett. 71, 2022-2025.

Lee, J.Y., Jeon, W., 2019. Exact solution of Euler-Bernoulli equation for acoustic black holes via generalized hypergeometric differential equation. J. Sound Vib. 452, 191-204.

Liang, Z., Li, J., 2012. Extreme acoustic metamaterial by coiling up space. Phys. Rev. Lett. 108, 114301.

Lin, S.-C.S., Huang, T.J., Sun, J.-H., Wu, T.-T., 2009. Gradient-index phononic crystals. Phys. Rev. B 79, 094302.

Liu, T., Chen, F., Liang, S., Gao, H., Zhu, J., 2019. Subwavelength Sound Focusing and Imaging Via Gradient Metasurface-Enabled Spoof Surface Acoustic Wave Modulation. Phys. Rev. Applied 11, 034061.

Liu, Y., Liang, Z., Liu, F., Diba, O., Lamb, A., Li, J., 2017. Source Illusion devices for flexural Lamb waves using elastic metasurfaces. Phys. Rev. Lett. 119, 034301.

Liu, Z., Zhang, X., Mao, Y., Zhu, Y.Y., Yang, Z., Chan, C.T., Sheng, P., 2000. Locally resonant sonic materials. Science 289, 1734-1736.

Lu, D., Liu, Z., 2012. Hyperlenses and metalenses for far-field super-resolution imaging. Nat. Commun. 3, 1205.

Ma, C., Escobar, M.A., Liu, Z., 2011. Extraordinary light focusing and Fourier transform properties of gradient-index metalenses. Phys. Rev. B 84, 195142.

Pelat, A., Gautier, F., Conlon, S.C., Semperlotti, F., 2020. The acoustic black hole: A review of theory and applications. J. Sound Vib. 476, 115316.

Pendry, J.B., 2000. Negative refracion makes a perfect lens. Phys. Rev. Lett. 85, 3966-3969.

Raguin, L., Gaiffe, O., Salut, R., Cote, J.M., Soumann, V., Laude, V., Khelif, A., Benchabane, S., 2019. Dipole states and coherent interaction in surface-acoustic-wave coupled phononic resonators. Nat. Commun. 10, 4583.

Rupin, M., Catheline, S., Roux, P., 2015. Super-resolution experiments on Lamb waves using a single emitter. Appl. Phys. Lett. 106, 024103.

Rupin, M., Lemoult, F., Lerosey, G., Roux, P., 2014. Experimental demonstration of ordered and disordered multiresonant metamaterials for lamb waves. Phys. Rev. Lett. 112, 234301.

Shen, Y.X., Peng, Y.G., Cai, F., Huang, K., Zhao, D.G., Qiu, C.W., Zheng, H., Zhu, X.F., 2019. Ultrasonic super-oscillation wave-packets with an acoustic meta-lens. Nat. Commun. 10, 3411.

Song, A., Li, J., Shen, C., Peng, X., Zhu, X., Chen, T., Cummer, S.A., 2019. Broadband high-index prism for asymmetric acoustic transmission. Appl. Phys. Lett. 114, 121902.

Sukhovich, A., Merheb, B., Muralidharan, K., Vasseur, J.O., Pennec, Y., Deymier, P.A., Page, J.H., 2009. Experimental and theoretical evidence for subwavelength imaging in phononic crystals. Phys. Rev. Lett. 102, 154301.

Tol, S., Degertekin, F.L., Erturk, A., 2016. Gradient-index phononic crystal lens-based enhancement of elastic wave energy harvesting. Appl. Phys. Lett. 109, 063902.

Verslegers, L., Catrysse, P.B., Yu, Z., Fan, S., 2009. Deep-subwavelength focusing and steering of light in an aperiodic metallic waveguide array. Phys. Rev. Lett. 103, 033902.

Wu, T.-T., Chen, Y.-T., Sun, J.-H., Lin, S.-C.S., Huang, T.J., 2011. Focusing of the lowest 
antisymmetric lamb wave in a gradient-index phononic crystal plate. Appl. Phys. Lett. 98, 171911. Xia, X., Yang, Q., Li, H., Ke, M., Peng, S., Qiu, C., Liu, Z., 2017. Acoustically driven particle delivery assisted by a graded grating plate. Appl. Phys. Lett. 111, 031903.

Zhao, J., Bonello, B., Boyko, O., 2016a. Focusing of the lowest-order antisymmetric Lamb mode behind a gradient-index acoustic metalens with local resonators. Phys. Rev. B 93, 174306.

Zhao, J., Pan, Y., Zhong, Z., 2013. A study of pressure-shear vertical wave propagation in periodically layered fluid and piezoelectric structure. J. Appl. Phys. 113, 054903.

Zhao, J., Yuan, W., Boyko, O., Bonello, B., Zhang, X., Pan, Y., Zhong, Z., 2019. Experimental evidence of quadrupolar whispering-gallery modes in phononic crystal based waveguides. AIP Adv. 9, 085032. Zhao, L., Conlon, S.C., Semperlotti, F., 2015. An experimental study of vibration based energy harvesting in dynamically tailored structures with embedded acoustic black holes. Smart Mater. Struct. 24, 065039.

Zhao, S.-D., Wang, Y.-S., Zhang, C., 2016b. Acoustic imaging and mirage effects with high transmittance in a periodically perforated metal slab. J. Appl. Phys. 120, 194901.

Zhou, X., Hu, G., 2011. Superlensing effect of an anisotropic metamaterial slab with near-zero dynamic mass. Appl. Phys. Lett. 98, 263510.

Zhu, X., Li, K., Zhang, P., Zhu, J., Zhang, J., Tian, C., Liu, S., 2016. Implementation of dispersion-free slow acoustic wave propagation and phase engineering with helical-structured metamaterials. Nat.

Commun. 7, 11731.

Zhu, Y., Hu, J., Fan, X., Yang, J., Liang, B., Zhu, X., Cheng, J., 2018. Fine manipulation of sound via lossy metamaterials with independent and arbitrary reflection amplitude and phase. Nat. Commun. 9 , 1632. 